\title{
O Hospital das Clinicas da Faculdade de Medicina de Ribeirão Preto da Universidade de São Paulo
}

\author{
Marcos Felipe Silva de Sá \\ Superintendente do HCRPFMRP-USP (1995-2002)
}

$\mathrm{O}$ Hospital das Clínicas da Faculdade de Medicina de Ribeirão Preto da Universidade de São Paulo foi idealizado no início da década de 50, para servir como campo de ensino e pesquisa para as recém-criadas Faculdade de Medicina e Escola de Enfermagem de Ribeirão Preto da Universidade de São Paulo.

A Faculdade de Medicina de Ribeirão Preto da Universidade de São Paulo, nos seus primórdios, desenvolvia as atividades clínicas, precariamente, nos pavilhões da Santa Casa de Misericórdia local. Naquela época, a Fundação Maternidade Sinhá Junqueira estava construindo uma maternidade à Rua Bernardino de Campos, um prédio de 4 andares com capacidade de 150 leitos. As obras ainda estavam em andamento, quando foi sugerida a idéia de entregá-la, por comodato, ao Estado de São Paulo, para que ali fosse instalado o Hospital das Clínicas da Faculdade de Medicina de Ribeirão Preto da USP.

D. Sinhá firmou convênio com a direção da Faculdade de Medicina de Ribeirão Preto-USP em 9 de abril de 1953 (Foto 1), pelo qual, durante vinte anos, a Maternidade Sinhá Junqueira integraria o sistema de ensino do curso médico, funcionando ali as clínicas obstétrica, ginecológica, médica e cirúrgica, até a construção definitiva do Hospital das Clínicas da Faculdade de Medicina em Monte Alegre, atual Campus Universitário da USP. Em contrapartida, a direção da FMRP-USP doou dois milhões de cruzeiros à Fundação Sinhá Junqueira para construção e instalação de uma casa de cultura na cidade.

Foram articuladores desse processo o Dr. Waldemar Barnsley Pessoa, Presidente da Fundação Maternidade Sinhá Junqueira; Dr. Paulo Gomes Romeo, Presidente do Centro Médico local e que, mais tarde, viria a ser o primeiro Superintendente do HCFMRP-USP (Foto 2), e o Prof. Zeferino Vaz, Diretor da FMRP-USP.

No ato de inauguração, em 11 de novembro de 1954, estiveram presentes o Governador Lucas Garcez e Sra., D. Carmelita Garcez; o Dr. Altino Arantes, ex-governador de São Paulo; o Prof. Melo Moraes, Reitor da USP; D. Sinhá Junqueira, Prof. Zeferino Vaz e outras autoridades civis, militares e representantes do clero.

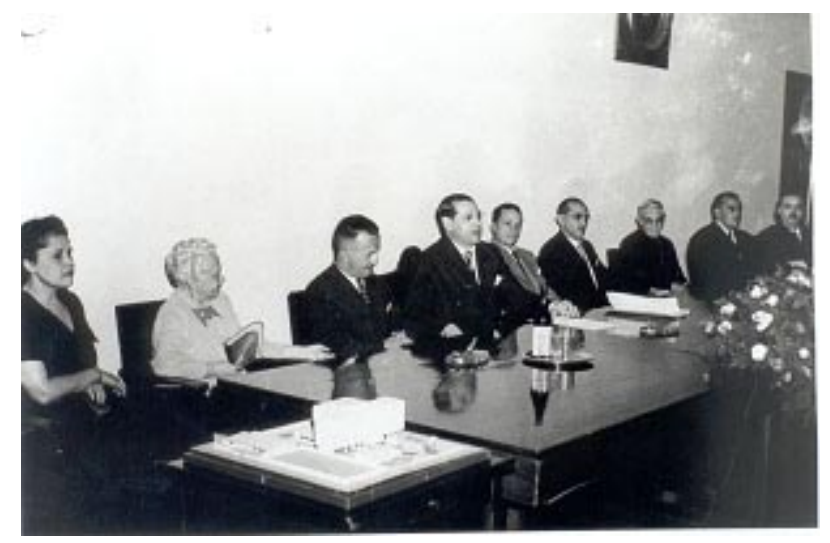

Foto 1 - Momento da assinatura do convênio entre a Universidade de São Paulo (FMRP) e a Fundação Maternidade "Sinhá Junqueira", para instalação e funcionamento do Hospital das Clínicas de Ribeirão Preto. Mesa formada por autoridades ribeirãopretanas (direita para esquerda): Sra. Glete Alcântara, Diretora da Escola de Enfermagem de Ribeirão Preto; Sra. Sinhá Junqueira; Prof.Dr. Zeferino Vaz, Diretor da Faculdade de Medicina de Ribeirão Preto; o Reitor da USP - Prof. Dr. Ernesto Leme; Cel. Condeixa - Prefeito de Ribeirão Preto; Sr. Domingos Cêntola - Presidente da Câmara Municipal; Monsenhor Laureano; Sr. Luiz Gonzaga Seixas e Dr. Paulo Gomes Romeo, Futuro Diretor Superintendente do Hospital das Clínicas de Ribeirão Preto / Fundação Maternidade "Sinhá Junqueira". 


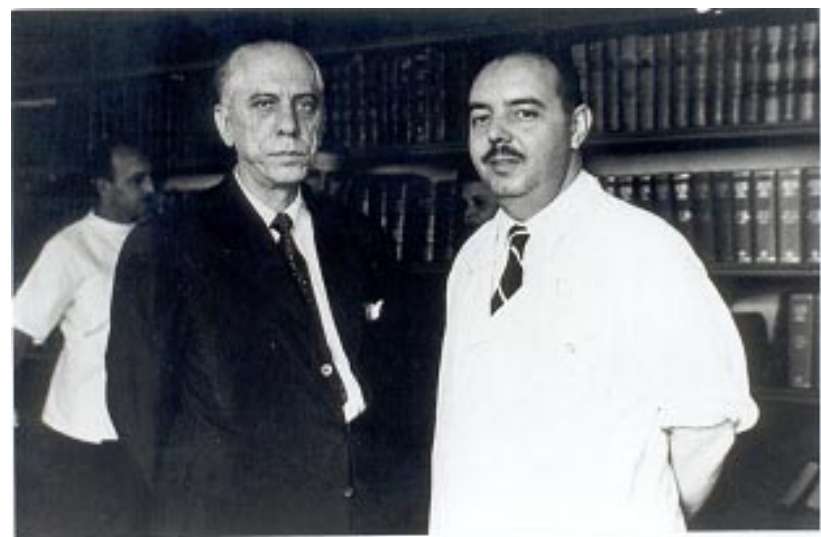

Foto 2 - Dr. Waldemar B. Pessoa (E) e Dr. Paulo Gomes Romeo.

Acertado o convênio entre a FMRP e a Fundação Sinhá Junqueira, o Prof. Zeferino Vaz deu início ao plano de conclusão da Maternidade Sinhá Junqueira, com as modificações que a prática e as novas finalidades vieram a exigir, inclusive com ampliação da área (Foto 3). Mais tarde, quando do término do comodato, o prédio viria a ser desapropriado pelo Estado de São Paulo, para dar lugar à atual Unidade de Emergência do HCFMRP-USP.
Naquele mesmo ano de 1954, já em 17 de setembro, o Diário Oficial do Estado de São Paulo havia publicado a mensagem n ${ }^{\circ} 297$ do Sr. Governador Lucas Nogueira Garcez, à Assembléia Legislativa, submetendo a ela o projeto de lei de criação do Hospital das Clínicas da Faculdade de Medicina de Ribeirão Preto, conforme texto a seguir.

Projeto de Lei n. 789, de 1954

Mensagem n. 297, do Sr. Governador do Estado à Assembléia Legislativa de São Paulo

São Paulo, 6 de setembro de 1954

"Senhor Presidente

Tenho a honra de, por intermédio de Vossa Excelência, submeter ao alto exame dessa nobre Assembléia o incluso projeto de lei que dispõe sobre a instituição, como entidade autárquica, do Hospital das Clínicas da Faculdade de Medicina de Ribeirão Preto e dá outras providências.

Instalada que foi a Faculdade de Medicina de Ribeirão Preto, cuidou-se desde logo, de tudo preparar a fim de, ao ser atingida a fase de ensino em que o doente é elemento essencial, contar a Faculdade com adequada e paralela organização hospitalar.

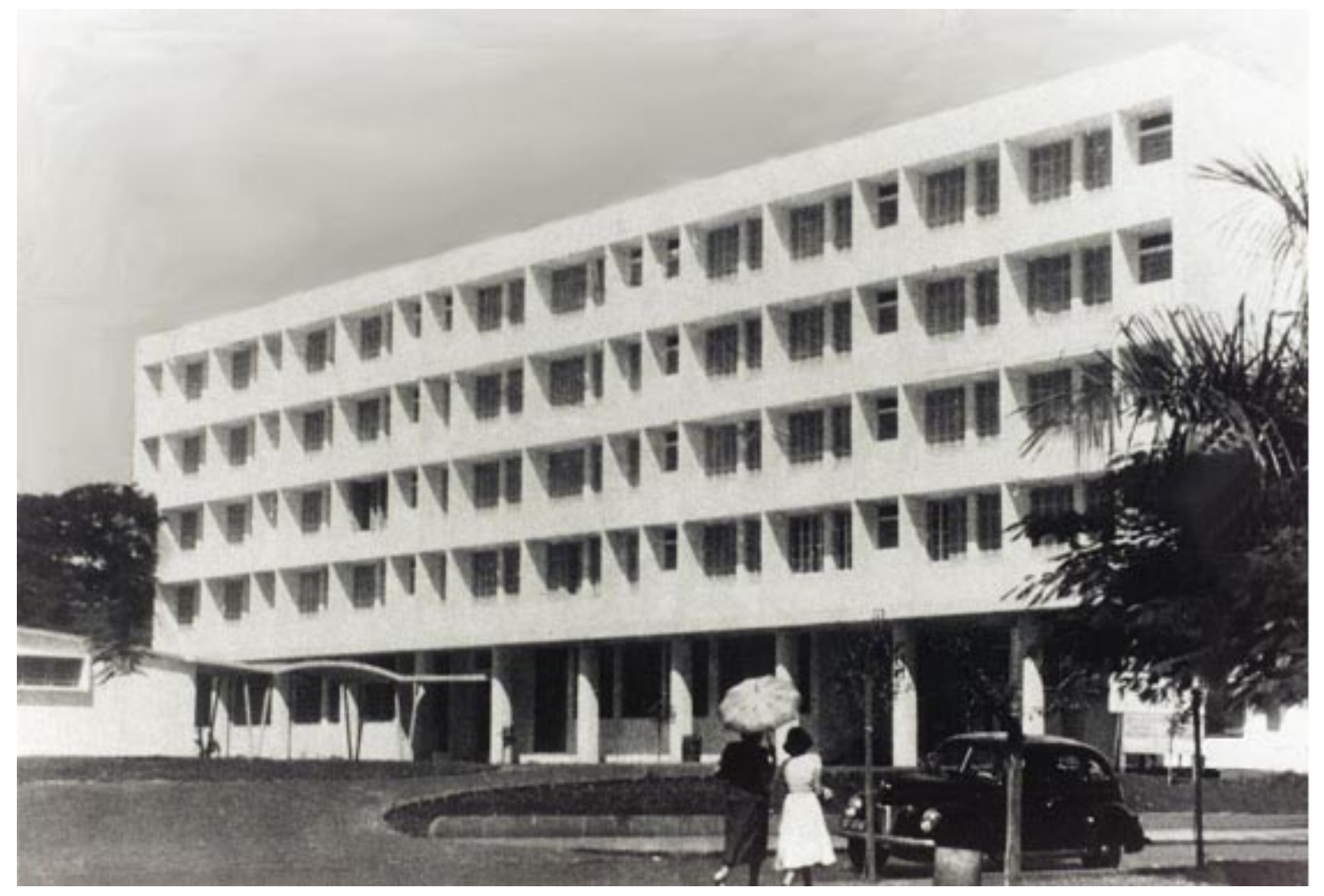

Foto 3 - Fachada do Hospital das Clínicas da Faculdade de Medicina de Ribeirão Preto da USP à época da inauguração. 
Assim, na forma do Convênio estabelecido com a Fundação "Sinhá Junqueira", foi cedido e transferido à Faculdade, pelo prazo de vinte anos, o terreno, edifício em construção e demais instalações da Maternidade "Sinhá Junqueira". Cuida-se, no momento, de terminar o edifício e de provê-lo dos elementos necessários para, dentro em breve, ser inaugurado.

Tendo em vista não ser possível, em curto espaço de tempo, construir o Estado o Hospital das Clínicas da Faculdade de Medicina de Ribeirão Preto, a Fundação "Sinhá Junqueira" concordou viessem a funcionar, provisoriamente, no edifício da Maternidade, ao invés da Escola de Enfermagem e Maternidade, as clínicas médica, cirúrgica e obstétrica, da Faculdade.

Dentro em breve, pois, será instalado, na Maternidade "Sinhá Junqueira", o Hospital das Clínicas da Faculdade de Medicina de Ribeirão Preto.

Para que, no momento oportuno, possa o Hospital ser posto em funcionamento, é que submeto a essa Casa o incluso projeto de lei que o institui em entidade autárquica, cria os cargos indispensáveis, prevê o regime de seu pessoal e dispõe sobre outras medidas correlatas.

Finalmente, cumpre salientar ser a organização, que o projeto objetiva dar ao Hospital de Ribeirão Preto, fruto da experiência já adquirida, pela Universidade, no Hospital das Clínicas de São Paulo.

Reitero a Vossa Excelência os protestos de minha alta consideração"

\section{LUCAS NOGUEIRA GARCEZ \\ Governador do Estado}

Em 24 de dezembro de 1955, foi publicada a lei $n^{\circ} 3274$, instituindo em entidade autárquica, o Hospital das Clínicas da Faculdade de Medicina de Ribeirão Preto da Universidade de São Paulo. As atividades do HCFMRP-USP iniciaram-se em 31 de junho de 1956 e, naquele ano, já foram feitas as primeiras internações e a primeira cirurgia.

Pouco tempo depois, foi adquirido, no quadrilátero defronte (atual Centro de Convenções Ribeirão Preto), o prédio de um antigo seminário, onde foram instalados os ambulatórios e alguns departamentos e serviços como Departamento de Oftalmologia e Otorrinolaringologia, Departamento de Medicina Social, serviço de registro de pacientes, arquivo médico, refeitório dos residentes e outros.

A lei de criação da Faculdade de Medicina de Ribeirão Preto-USP previa que os integrantes do cor- po docente, todos em RDIDP, exercessem atividades na chamada Clínica Civil. Como as instalações eram precárias e os espaços reduzidos, eram permitidas internações no Hospital São Francisco.

Com o passar do tempo, cresceu a necessidade da construção de uma nova sede para o Hospital das Clínicas, que atendesse não só à demanda de pacientes, mas que, sobretudo, propiciasse ao corpo docente e discente da Faculdade de Medicina de Ribeirão Preto e da Escola de Enfermagem condições confortáveis para o exercício das atividades de ensino, pesquisa e assistência.

Assim, em 1962, foi assinado o contrato para a elaboração de um projeto que daria sede própria ao HCRP no Campus da USP, com capacidade prevista de 500 leitos (Foto 4). As obras tiveram interrupções, mas, finalmente, em 1978, o Hospital das Clínicas inaugurou sua sede no Campus da USP, integrando os Departamentos Básicos e Clínicos (Foto 5).

Naquela época, foram disponibilizados 525 leitos. O número de consultas era, em média, 700/dia. O antigo prédio da cidade ficou reservado para a instalação de uma Unidade de Atendimento de Urgências (hoje Unidade de Emergência).

Vale ressaltar que, ao longo da sua história, o Hospital das Clínicas da FMRP-USP ganhou destaque por alguns acontecimentos pioneiros ou marcantes para

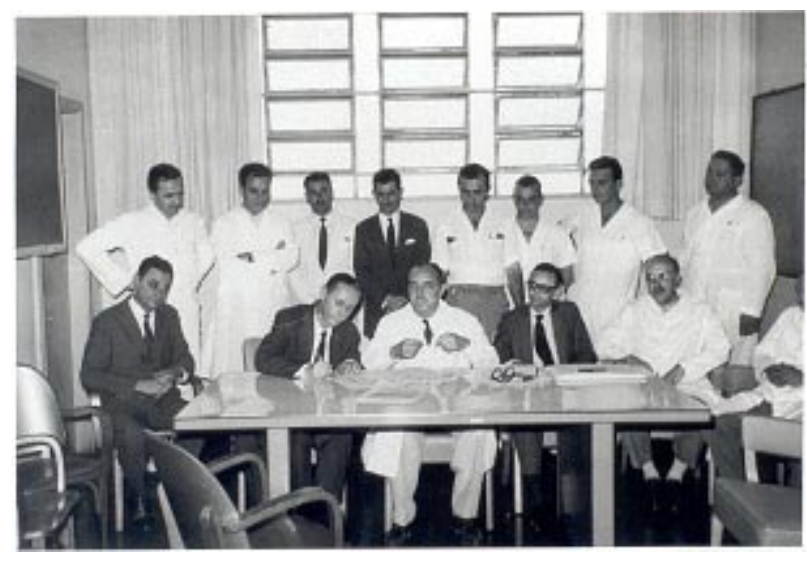

Foto 4 - Momento da assinatura do contrato firmado entre o HCRP e a FOMISA, presentes: Direção do HCRP e FOMISA (engenheiros) para planejamento da construção do Hospital das Clínicas da Faculdade de Medicina de Ribeirão Preto da USP. Da direita para esquerda: (em pé) Prof. Dr. Carlos Eduardo Martinelli; Sérgio P. Silva Lisboa; Sylvio Bonfá; Eng ${ }^{\circ}$ Maomed Cozac; Adolpho Ubida; Dr. Nagib Scaff; Prof. Almiro P. Azeredo; Prof. Hernan Davanzo Corte. Sentados: Prof.Dr. Jorge Armbrust de Lima Figueiredo; Prof.Dr. Ruy Escorel Ferreira-Santos; Arquiteto Oscar Valdetaro; Dr. Paulo Gomes Romeo - Superintendente do HCRP; Edwin Karen - Gerente da FOMISA; Dr. Jorge Gabriel Borba - Consultor da FOMISA. 


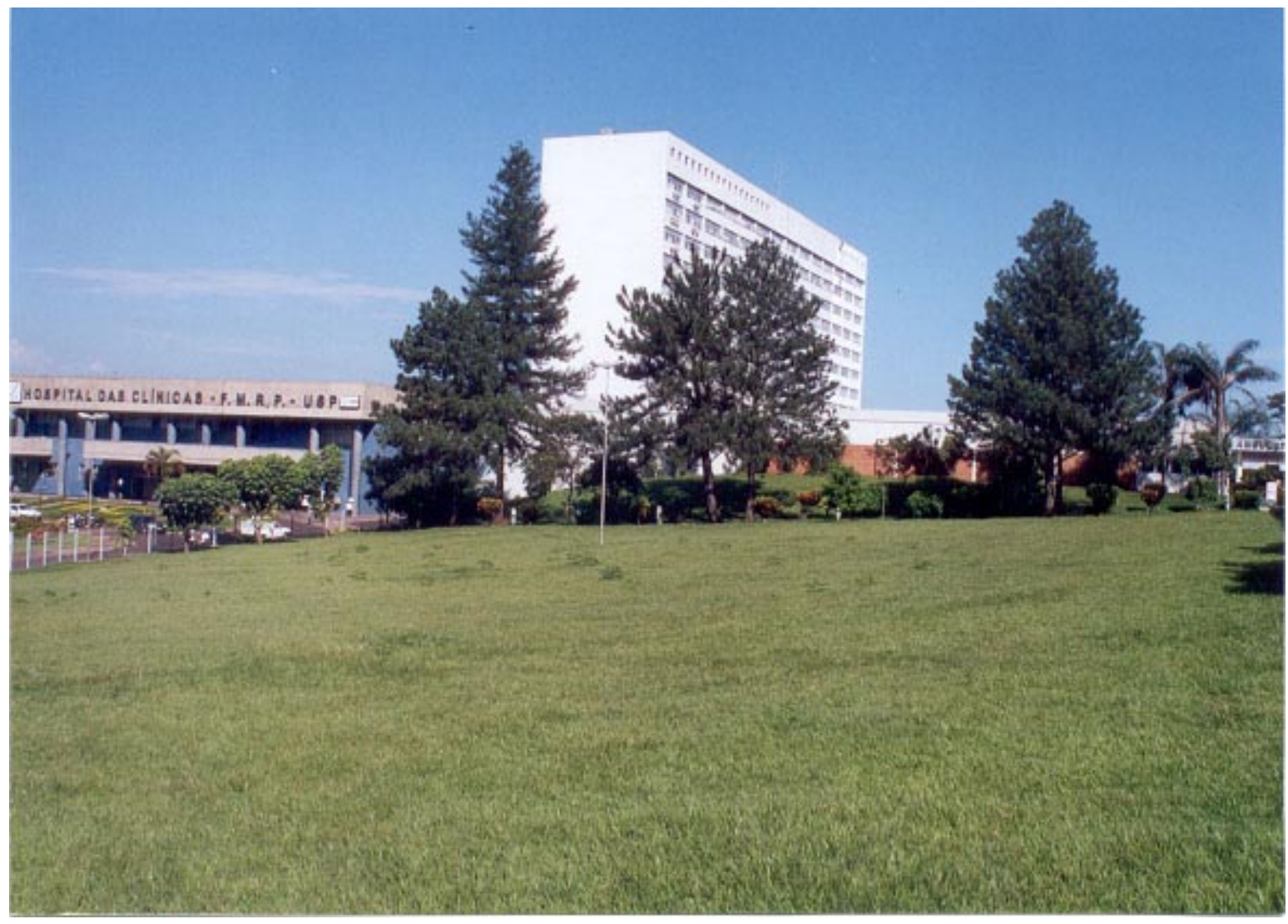

Foto 5 - Hospital das Clínicas da FMRP-USP - Campus da USP.

a Medicina Brasileira, colocando Ribeirão Preto no rol dos grandes centros médicos do país. O HCFMRPUSP é hoje Centro de Referência do Ministério da Saúde para várias áreas da Medicina, dentre as quais destacamos: Transplantes de Rim, Fígado, Medula Óssea e Córnea; Cirurgia de Epilepsia; Gestação de Alto Risco; Neurocirurgia; Cardiologia Clínica e Cirúrgica; Oftalmologia - Banco de Olhos; Doenças Infecciosas - AIDS; Centro de Queimados; Hemoterapia - Hemocentro; Otorrinolaringologia - Implante Coclear; Oncologia; Imunobiológicos Especiais; Erros Inatos do Metabolismo ("teste do pezinho"); Banco de Leite Humano; Reabilitação e Dermatologia Sanitária.

O grau de informatização do HCFMRP-USP atingiu patamares superiores aos dos demais hospitais brasileiros. A implantação da prescrição informatizada o coloca entre os $5 \%$ dos hospitais, no mundo, que têm essa tecnologia implementada, e é o único hospital público do país a ter prescrição eletrônica $100 \%$ implementada.

Em número de atendimento, o HCRP apresenta, hoje, estatísticas impressionantes.
HCFMRP-USP - Dados de 2001

- $\mathrm{N}^{\mathrm{o}}$ de leitos - 852

- $\mathrm{N}^{\mathrm{o}}$ de internações/ano - 33.214

- $\mathrm{N}^{\mathrm{o}}$ de consultas/ano - 546.062

- Exames de laboratório/ano - 2.030.851

- Exames complementares procedimentos especializados/ano - 402.799

- Cirurgias/ano - 27.310

- $\mathrm{N}^{\circ}$ de servidores - 4.309

Com a criação do SUS, em 1988, uma nova concepção de Serviço Público de Saúde foi implantada no Brasil. A ordenação hierarquizada do fluxo de pacientes fez com que a demanda por serviços especializados, na região fosse se concentrando no Hospital das Clínicas, tornando-o referência terciária regional dentro do novo sistema.

O Hospital das Clínicas vem cumprindo rigorosamente seu papel dentro do SUS. Tem tomado iniciativas pioneiras, no sentido de organizar o fluxo de pacientes, interagindo de maneira ativa com os gestores municipais e regionais da saúde. Assim, a partir de janeiro de 2000, duas providências fundamentais fo- 
ram tomadas neste sentido: 1) A descentralização do agendamento de pacientes para consultas eletivas no HC Campus, hoje $100 \%$ informatizada e controlada pelas Direções Regionais de Saúde (DIRs); 2) Criação da Central Única de Regulação Médica (CURM) para Emergências, em parceria com a DIR XVIII (Ribeirão Preto) e Secretaria Municipal da Saúde, o que possibilitou ordenar de maneira criteriosa o fluxo de pacientes em situação de emergência. Esse modelo assistencial tornou nossa região referência para os gestores da saúde, de todo o Brasil.

Do ponto de vista de formação de recursos humanos, além de ser o campo de ensino para os alunos de graduação da Faculdade de Medicina de Ribeirão Preto e Escola de Enfermagem de Ribeirão Preto da USP, o Hospital das Clínicas de Ribeirão Preto tem concentrado esforços na expansão dos programas de pós-graduação stricto e lato sensu.

Centenas de médicos são recebidos anualmente para programas de residência, aperfeiçoamento, especialização e reciclagem (atualização). Para os demais profissionais da saúde existem 27 programas de aprimoramento em 11 áreas de atuação (Psicologia, Nutrição, Fisioterapia, Terapia Ocupacional, Farmácia, Laboratórios de Análises Clínicas, Serviço Social, Fonoaudiologia, Física Médica, Saúde Comunitária e Enfermagem).

Os departamentos clínicos da FMRP-USP têm, hoje, um dos maiores programas de pós-graduação stricto sensu do país na área médica. Em 2001, havia 645 alunos matriculados em 11 diferentes áreas desenvolvidas no HCFMRP-USP. Esses programas têm recebido os melhores conceitos de avaliação pela CAPES.

Para organização dos diferentes programas de ensino foi criado, em 1998, o Centro de Educação e Aperfeiçoamento Profissional em Saúde (CEAPS). O CEAPS dispõe de infra-estrutura burocrática e áreas de apoio (anfiteatro, salas de aula, bibliotecas, etc.) para cursos e eventos. À exceção dos Cursos de Graduação, Pós-Graduação Stricto Sensu e Residência Médica, todos os demais programas de aperfeiçoamento, especialização e aprimoramento são centralizados no CEAPS. Somente no ano 2001 foram promovidos 657 eventos entre cursos, jornadas, simpósios, aulas, palestras e outros.

Estão colocados à disposição do corpo docente da FMRP-USP, no HC Campus, cerca de 6 anfiteatros, 35 salas de aula, 10 salas de reuniões, totalizando 1650 assentos. Na Unidade de Emergência, estão disponíveis 3 anfiteatros com capacidade para 342 luga- res, além do Centro de Estudos em Emergência em Saúde (CEES), que dispõe de sala de aula, salas de reuniões, salas de estudos e biblioteca.

O Centro Interescolar do HCFMRP-USP, vinculado ao CEAPS, hoje, dispõe de instalações próprias e é um dos maiores centros formadores de recursos humanos de nível médio para a rede pública e privada de saúde. São oferecidos cursos para auxiliares de enfermagem, técnicos de enfermagem, técnicos de radiologia, técnicos de laboratórios clínicos, etc. (Foto 6)

No que diz respeito à pesquisa, o HCFMRPUSP coloca à disposição dos pesquisadores da FMRP e EERP da USP, além de seus ambulatórios, enfermarias, salas cirúrgicas e laboratórios, invejável Centro de Cirurgia Experimental e as modernas instalações do Hemocentro, para que possam desenvolver seus projetos de pesquisa. Somente no ano de 2001, foram aprovados pelo Comitê de Ética em Pesquisa do HCRP 249 projetos. Foram defendidas 163 dissertações de mestrado e 134 teses de doutorado, desenvolvidas no HCFMRP pelos alunos de Pós-Graduação da Faculdade de Medicina de Ribeirão Preto-USP e Escola de Enfermagem de Ribeirão Preto da USP e publicados 770 trabalhos, em revistas nacionais e internacionais, vários deles premiados, oriundos das pesquisas ali desenvolvidas.

Para acompanhar o ritmo da modernização tecnológica e da agilidade gerencial nos dias atuais, foi fundamental a criação da Fundação de Apoio ao Ensino, Pesquisa e Assistência - FAEPA - do Hospital das Clínicas de Ribeirão Preto, em 1988. A FAEPA tem propiciado ao HCFMRP-USP condições para o desenvolvimento de Recursos Humanos para atender demandas administrativas e de assistência, assim como o gerenciamento dos recursos para os investimentos em equipamentos e adequação da estrutura física para melhor conforto dos usuários.

Através da FAEPA foi possível viabilizar a construção do Centro de Convenções Ribeirão Preto (Foto 7), disponibilizando para Ribeirão Preto e Região, avançada infra-estrutura, com padrão de excelência internacional e instalações que abrangem uma área de $15.000 \mathrm{~m}^{2}$, proporcionando total conforto e comodidade em até 2.500 lugares, com todos os recursos de áudio e vídeo, para a realização de pequenos, médios e grandes eventos.

Enfim, falar sobre o HCFMRP-USP é falar sobre a saúde pública de Ribeirão Preto e Região. Nos seus 46 anos de existência, o HCRP viveu intensamente muitos aspectos que envolvem a questão da 


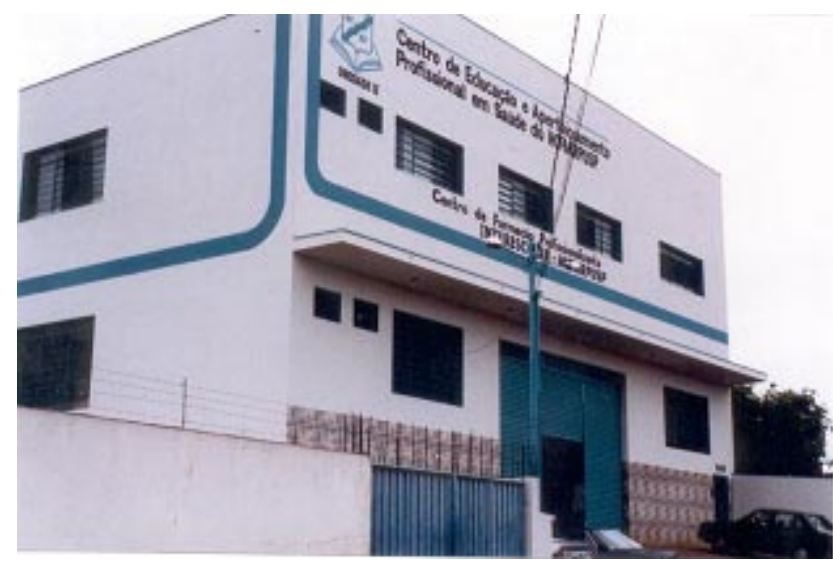

Foto 6 - Centro de Formação Profissionalizante - Interescolar do HCFMRP-USP, à Rua Aquidauana, 1049.

saúde no país, mas nem os momentos mais críticos da saúde pública brasileira conseguiram fazer com que o HCRP deixasse de ser o maior hospital do interior do Brasil, reconhecido e respeitado no país e no exterior. Isso só permanece possível graças à verdadeira abnegação, ao espírito solidário e à coragem que todos apresentam nos momentos difíceis e na superação das situações mais críticas. A forma responsável com que o HCFMRP-USP desenvolve suas atividades dá mostra de que é uma instituição que tem consciência das

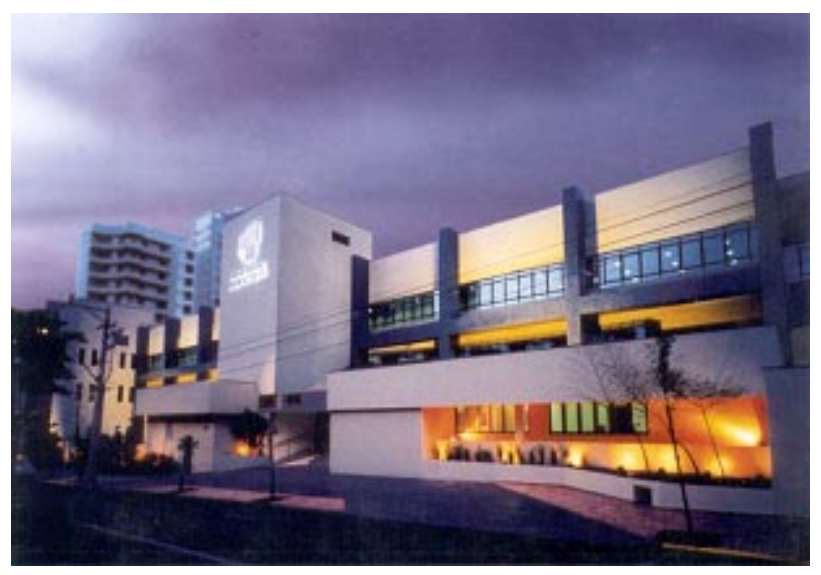

Foto 7 - Centro de Convenções Ribeirão Preto da Fundação de Apoio ao Ensino, Pesquisa e Assistência do HCFMRP-USP (FAEPA), à Rua Bernardino de Campos, 999.

dimensões de seu papel no contexto da saúde pública nacional.

\section{AGRADECIMENTOS}

O autor agradece as pessoas que colaboraram para elaboração deste texto: Dr. Carlos Eduardo Martinelli, Sra. Geralda Silva Romeo, Dr. Luiz Scatena, Sra. Ódan Maria Nunes, Sra. Susana Gomes Romeo e Maria Teresa Arosti Stocco. 